\title{
Remoção cirúrgica de odontoma composto em paciente pediátrico: relato de caso
}

Surgical removal of compound odontoma in pediatric patients: case report

Remoción quirúrgica de odontoma compuesto en paciente pediátrico: reporte de caso

\author{
Silvestre Estrela da SILVA JÚNIOR' \\ Lukas Natã Mendes FRAGOSO' ${ }^{1}$ \\ Nathalia da Cruz FLORES 1 \\ Laís dos Santos NOVAIS 1
}

Maria Vitória Calado Ramalho dos SANTOS ${ }^{1}$

Bruna Landim PINHEIRO1

Vicente Jadson Gregorio FREITAS ${ }^{1}$

Fátima Roneiva Alves FONSECA ${ }^{2}$

Eduardo Dias RIBEIRO ${ }^{3}$

Julierme Ferreira ROCHA

${ }^{1}$ Departamento de Odontologia do Centro de Saúde e Tecnologia Rural, Universidade Federal de Campina Grande, UFCG, 58708-110 Patos, Paraíba, Brasil ${ }^{2}$ Departamento de Ortodontia e Desenvolvimento Craniofacial, Universidade Federal de Campina Grande, UFCG, 58708-110 Patos, Paraíba, Brasil

${ }^{3}$ Departamento de Cirurgia e Traumatologia Bucomaxilofacial, Universidade Federal da Paraíba - UFPB Campus I - Cidade Universitária $58051-900$ João Pessoa - PB, Brasil ${ }_{4}^{4}$ Departamento de Cirurgia e Traumatologia Bucomaxilofacial, Universidade Federal de Campina Grande, UFCG, 58708-110 Patos, Paraíba, Brasil

\section{Resumo}

Introdução: Os odontomas são tumores odontogênicos benignos e os mais frequentes dos maxilares. Usualmente estão associados à dentição permanente em crianças e adultos jovens, sendo geralmente assintomáticos, podendo causar impactação dental. O aspecto radiográfico é de múltiplas calcificações, semelhantes ao dente, circundadas por um estreito halo radiolúcido, sendo o diagnóstico feito através de exames radiográficos de rotina. Objetivo: Este trabalho teve como objetivo relatar a remoção cirúrgica de um odontoma composto em região anterior da maxila, associado a dentes impactados, em um paciente pediátrico. Relato do caso clínico: Paciente do sexo masculino, 12 anos, foi referido ao serviço de cirurgia oral da Universidade Federal de Campina Grande, Campus Patos - PB, encaminhado pelo ortodontista. Durante anamnese não foram constatados comprometimento sistêmico. Ao exame físico foi observado abaulamento na região maxilar esquerda e retenção prolongada de alguns elementos, como também a ausência de outros. Analisada a radiografia panorâmica, foi observada massa radiopaca, na região anterior da maxila, sugestiva de odontoma composto, com impactação dental. Em decorrência da proximidade da lesão com os dentes anteriores superiores impactados e da localização do elemento 21, foi feita tomografia computadorizada por feixe cônico, o que facilitou o planejamento cirúrgico. O procedimento foi realizado sem intercorrências e no pós-operatório tardio, o paciente evolui satisfatoriamente. Conclusão: Pode-se concluir que o tratamento proposto foi eficaz e que a tomografia computadorizada por feixe cônico é um exame complementar de grande valia no diagnóstico de patologias, assim como no planejamento cirúrgico, devido à alta resolução e precisão das imagens obtidas.

Descritores: Tumores Odontogênicos; Odontoma; Tomografia Computadorizada de Feixe Cônico.

\section{Abstract}

Introduction: Odontomas are benign odontogenic tumors and the most frequent tumors of the jaw. They are usually associated with permanent dentition in children and young adults, and are generally asymptomatic and may cause dental impingement. The radiographic aspect is of multiple calcifications, similar to the tooth, surrounded by a narrow radiolucent halo, thus, the diagnosis is made through routine radiographic examinations. Objective: The objective of this study is to report a clinical case of a composite odontoma in the maxilla, the surgical planning of which was done using a CT scan. Clinical case report: A 12-year-old male patient was referred to the oral surgery service of the Federal University of Campina Grande, Campus Patos - PB, by the orthodontist. After anamnesis and clinical examination, the panoramic radiograph was analyzed, and a radiopaque mass was observed in the anterior region of the maxilla, suggestive of compound odontoma, with dental impactation. Due to the proximity of the lesion with the impacted upper anterior teeth and the location of the element 21, a CT scan was performed with a conical bundle, which facilitated the surgical planning. The procedure was performed without trans and postoperative complications, the patient is progressing satisfactorily. Conclusion: Early diagnosis and intervention of jaw injuries is very important in the future rehabilitation of patients. Cone-beam computed tomography is a complementary exam of great value in the diagnosis of pathologies, as well as in surgical planning, due to the high resolution and accuracy of the images obtained.

Descriptors: Odontogenic Tumors; Odontoma; Cone-Beam Computed Tomography.

\section{Resumen}

Introducción: Los odontomas son tumores odontogénicos benignos y los más frecuentes de los maxilares. Usualmente están asociados a la dentición permanente en niños y adultos jóvenes, siendo generalmente asintomáticos, pudiendo causar impactación dental. El aspecto radiográfico es de múltiples calcificaciones, semejantes al diente, circundadas por un estrecho halo radiolúcido, siendo el diagnóstico hecho a través de exámenes radiográficos de rutina. Objetivo: Este trabajo tuvo como objetivo relatar la remoción quirúrgica de un odontoma compuesto en región anterior de la mandíbula, asociado a dientes impactados, en un paciente pediátrico. Relato de caso clínico: El paciente de sexo masculino, de 12 años, fue referido al servicio de cirugía oral de la Universidad Federal de Campina Grande, Campus Patos - PB, encaminado por el ortodoncista. Durante la anamnesis no se observó un compromiso sistémico. En el examen físico se observó abombamiento en la región maxilar izquierda y retención prolongada de algunos elementos, así como la ausencia de otros. Se analizó la radiografía panorámica, se observó masa radiopaca, en la región anterior de la mandíbula, sugestiva de odontoma compuesto, con impacto dental. En consecuencia de la proximidad de la lesión con los dientes anteriores superiores impactados y de la localización del elemento 21, se hizo tomografía computarizada por haz cónico, lo que facilitó la planificación quirúrgica. El procedimiento se realizó sin intercurrencias y en el postoperatorio tardío, el paciente evoluciona satisfactoriamente. Conclusión: Se puede concluir que el tratamiento propuesto fue eficaz y que la tomografía computarizada por haz cónico es un examen complementario de gran valor en el diagnóstico de patologías, así como en la planificación quirúrgica, debido a la alta resolución y precisión de las imágenes obtenidas

Descriptores: Tumores Odontogénicos; Odontoma; Tomografía Computarizada de Haz Cónico.

\section{INTRODUÇÃO}

O odontoma é uma malformação focal de origem odontogênica, semelhante a um tumor benigno, mas que não é classificado como uma neoplasia ${ }^{1}$. Dentre os tumores odontogênicos é o tipo mais comum, acometendo a população pediátrica na maioria dos casos ${ }^{2}$.

Em 1992 o odontoma foi classificado pela Organização Mundial da Saúde em dois tipos: 
composto e complexo. Ambos os tipos são malformações de tecidos dentários, sendo que no odontoma composto, o tipo mais comum, esses tecidos estão organizados de uma forma que se assemelham à pequenos dentes e no odontoma complexo eles se distribuem de forma desorganizada. Por vezes, a lesão pode apresentar os dois tipos, sendo classificada como mista ${ }^{3}$.

Geralmente os odontomas são assintomáticos e podem estar associados a um dente impactado, provocando distúrbios na erupção dentária que caracterizam o sinal clínico que geralmente estimula a procura pelo atendimento odontológico ${ }^{2}$.

O odontoma composto e complexo podem ser encontrados em qualquer região dos maxilares, porém o composto, tem uma maior prevalência e apresenta um potencial de crescimento menor, maior incidência na região anterior da maxila, sendo o dente canino o mais associado ${ }^{4}$.

Devido à ausência de sintomatologia, os odontomas são comumente diagnosticados através de exames radiográficos de rotina, geralmente na segunda década de vida. O odontoma composto totalmente desenvolvido caracteriza-se radiograficamente por múltiplas radiopacidades, semelhantes a pequenos dentículos, dentro de uma área radiolúcida de margens bem definidas ${ }^{5}$.

$\mathrm{O}$ diagnóstico e tratamento precoce do odontoma associado a um dente impactado aumentam as chances de preservação desse dente. Geralmente o tratamento consiste na enucleação simples seguida de curetagem, posteriormente pode ser necessário um segundo procedimento cirúrgico para o tracionamento do dente impactado ${ }^{2}$.

O objetivo desse trabalho foi relatar a remoção cirúrgica de um odontoma composto, associado a dentes impactados, em um paciente pediátrico.

\section{CASO CLÍNICO}

Paciente do sexo masculino, melanoderma, 12 anos de idade, foi encaminhado para a Clínica Escola de Odontologia da Universidade Federal de Campina Grande, Patos, Paraíba, Brasil para avaliação de retenção prolongada de decíduos, ausências dentárias e abaulamento ósseo localizado entre o incisivo central decíduo e canino decíduo superior esquerdo, diagnosticada durante uma consulta de rotina. Durante a anamnese, o paciente não relatou sintomatologia dolorosa na região e não foram constatados comprometimento sistêmico. Ao exame físico, observou-se abaulamento ósseo vestibular recoberto por tecido mole de consistência normal, ausência dos elementos 13, 21, 22 e 23; além de retenção prolongada dos elementos 53, 61, 62 e 63. Durante análise da radiografia panorâmica, a mesma evidenciou estruturas radiopacas, semelhante a dentes, circunscritas por um halo radiolúcido, sugestivo de odontoma composto, além da impactação dos elementos 13, 21 e 23 (Figura 1). Por esse exame de imagem, não podemos observar a presença do elemento 22 .

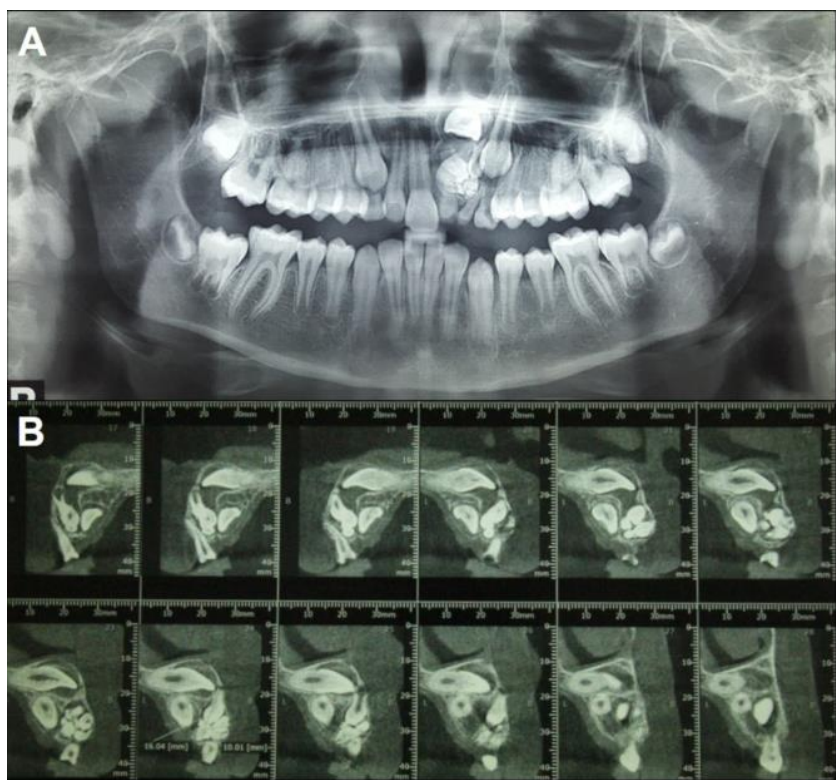

Figura 1: A - Radiografia panorâmica evidenciando estruturas radiopacas semelhantes a dentes na região anterior da maxila entre os elementos 61 e 63 . B - Corte tomográfico parassagital evidenciando localização do elemento 21 e tamanho da lesão.

Em decorrência da proximidade da lesão com os dentes anteriores superiores impactados, foi solicitado ao paciente a realização de uma tomografia computadorizada por feixe cônico confirmando a existência do elemento 22, a intima relação do elemento $21 \mathrm{com}$ o assoalho da fossa nasal e para planejamento cirúrgico. $\mathrm{O}$ tratamento proposto foi a remoção dos dentes $51,61,62,63,21$ e 22 que se apresentavam em intima relação com a lesão, sem possibilidade de tracionamento, além de enucleação cirúrgica conservadora da lesão. Uma hora antes da cirurgia o paciente foi medicado com uma dose única de dexametasona $4 \mathrm{mg}$ (dois comprimidos, totalizando $8 \mathrm{mg}$ ). Foi realizada antissepsia intraoral e extra oral com bochecho de Digluconato de Clorexina $0,12 \%$ não alcoólica (RioHex ${ }^{\circledR}$ RioQuímica® Indústria Farmacêutica Ltda, São José do Rio Preto - SP, Brasil) por 40 segundos e aplicação tópica de Digluconato de Clorexidina 2\% (RioHex® - RioQuímica ${ }^{\circledR}$ Indústria Farmacêutica Ltda, São José do Rio Preto - SP, Brasil) na região perioral, respectivamente. $\mathrm{O}$ procedimento foi iniciado através do bloqueio do nervo alveolar superior anterior e infraorbital esquerdo, além de bloqueio do nervo nasopalatino. Primeiramente estendendeu-se o lábio superior, do lado anestesiado, fazendo com que os tecidos da região da mucosa sejam afastados superiormente e a prega muco vestibular seja evidenciada, com uma seringa carpule com refluxo (DUFLEX® - SSWhite Artigos Dentários Ltda, Rio de Janeiro-RJ, Brasil), introduziu-se a agulha longa de $32 \mathrm{~mm}$ e $25 \mathrm{G}$ de calibre (UNOJECT® - DFL Indústria e Comércio 
Ltda, Rio de Janeiro-RJ, Brasil) na mucosa da face vestibular da maxila esquerda, na altura da prega muco vestibular diretamente sobre o $1^{\circ}$ pré-molar superior esquerdo, introduzindo a agulha paralela ao longo eixo do dente, indo em direção a área alvo que é o forame infraorbitário. Foi realizada aspiração, apresentando-se negativa, então foi injetado lentamente (cerca de 60 segundos) um tubete de $1,8 \mathrm{~mL}$ contendo a solução anestésica de Cloridrato de Articaína 4\% + Epinefrina 1:100.000 (ARTICAINE® - DFL Indústria e Comércio Ltda, Rio de Janeiro-RJ, Brasil). Retirando a agulha com um lento movimento ${ }^{6}$. Posteriormente, foi realizada anestesia do nervo infraorbital, onde foi depositado $0,9 \mathrm{~mL}$ do mesmo sal anestésico utilizado anteriormente. Logo após, foi realizada a anestesia do nervo nasopalatino, onde com auxílio de uma seringa carpule com refluxo (DUFLEX® - SSWhite Artigos Dentários Ltda, Rio de Janeiro-RJ, Brasil), introduziu-se a agulha longa de $32 \mathrm{~mm}$ e $25 \mathrm{G}$ de calibre (UNOJECT® - DFL Indústria e Comércio Ltda, Rio de Janeiro-RJ, Brasil) na mucosa palatina, na reigião da papila incisiva, tento como área alvo, o forame incisivo. Foi realizada aspiração, apresentando-se negativa, então foi injetado lentamente (cerca de 60 segundos) depositando $0,9 \mathrm{~mL} \mathrm{Da}$ solução anestésica de Cloridrato de Articaína $\quad 4 \%+$ Epinefrina $1: 100.000$ (ARTICAINE® - DFL Indústria e Comércio Ltda, Rio de Janeiro-RJ, Brasil). Retirando a agulha lentamente $^{6}$. Por fim, foram realizadas anestesias terminais infiltrativas ao redor da lesão e no ápice do elemento 53, com o auxílio de uma seringa carpule com refluxo (DUFLEX® - SSWhite Artigos Dentários Ltda, Rio de Janeiro-RJ, Brasil) e agulha longa de $32 \mathrm{~mm}$ e $25 \mathrm{G}$ de calibre (UNOJECT ${ }^{\circledR}$ - DFL Indústria e Comércio Ltda, Rio de Janeiro-RJ, Brasil), depositando $0,4 \mathrm{~mL}$ em três pontos ao redor da área cirúrgica, $0,9 \mathrm{~mL}$ no ápice do elemento $53 \mathrm{e}$ infiltração local nas papilas gengivais dos elementos envolvidos na região da abertura do retalho a fim de promover hemostasia, essa anestesia busca promover uma melhor hemostasia durante o procedimento cirúrgico e com isso melhorar o campo visual do operador. Durante todos os passos, manteve-se a comunicação com o paciente, explicando-lhe o procedimento $^{6}$. Para o acesso ao odontoma, foi feito o tracionamento do tecido mole do lábio superior com auxílio de um afastador do tipo Minessota (QUINELATO® - Schobell Industrial Ltda, Rio Claro - SP) a fim de estabelecer uma melhor visualização do campo cirúrgico. Com um cabo de bisturi $\mathrm{n}^{\circ} 3$ (QUINELATO® - Schobell Industrial Ltda, Rio Claro - SP) com lâmina $\mathrm{n}^{\circ} 15$ acoplada (EMBRAMAC $^{\Theta}$ - Indústria, Comércio Importação e Exportação Ltda, Itapira-SP, Brasil), foi confeccionado um retalho biangular baixo, em espessura total, entendendo-se do elemento 11 ao elemento 24. Logo depois, com o auxílio de um descolador do tipo Molt $\mathrm{n}^{\circ} 9$ (QUINELATO ${ }^{\circledR}$ Schobell Industrial Ltda, Rio Claro - SP) foi feito o descolamento mucoperiosteal da região supracitada (Figura 2).

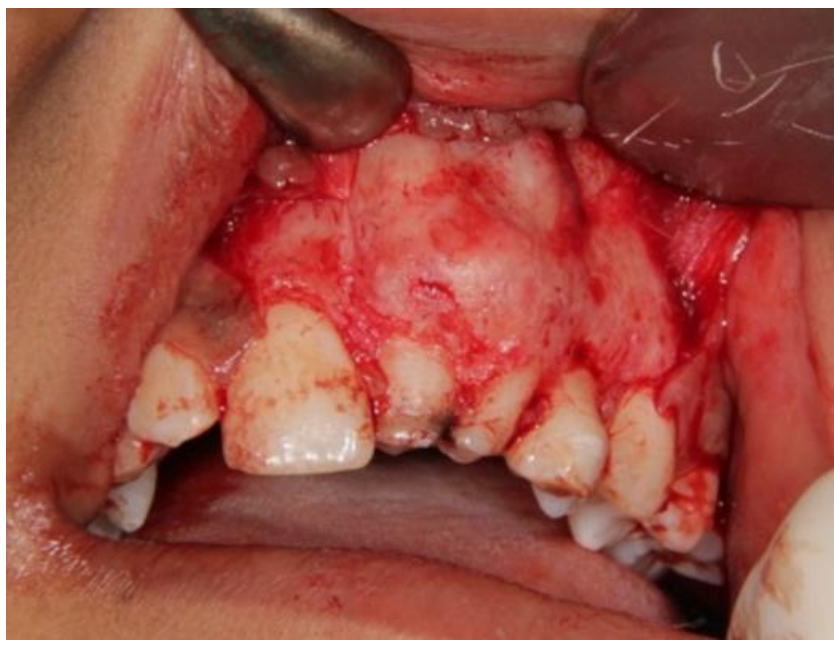

Figura 2: Aspecto trans-operatório da lesão.

Munido de um fórceps número 150 (QUINELATO® - Schobell Industrial Ltda, Rio Claro - SP), realizamos a luxação e remoção dos elementos 53, 61, 62 e 63 respectivamente. Realizado o acesso da região anterior da maxila, foi executada a técnica de osteotomia com o auxílio de motor de alta rotação (Kavo® do Brasil Indústria e Comercio Ltda, Joinville- SC) e broca cirúrgica esférica $\mathrm{n}^{\circ} 6$ (Microdont ${ }^{\circledR}$ Comércio, Importação e Exportação de Produtos para Uso Médico e Odontológico Ltda, São Paulo- SP) até obter visualização dos dentículos e abertura suficiente para saída dos mesmos, quando necessário, foi executada a técnica de odontossecção dos dentículos com o auxílio de motor de alta rotação e broca cirúrgica tronco-cônica $\mathrm{n}^{\circ} 702$ (Microdont ${ }^{\circledR}$ Comércio, Importação e Exportação de Produtos para Uso Médico e Odontológico Ltda, São Paulo- SP) a fim de facilitar sua saída da loja cirúrgica. Ao ponto de visualização dos elementos 21 e 22 , foi executada a técnica de odontossecção dos elementos com o auxílio de motor de alta rotação e broca cirúrgica tronco-cônica $\mathrm{n}^{\circ} 702$ a fim de facilitar a remoção dos elementos, onde os mesmos foram seccionados, inicialmente, em duas partes (coroa e raiz) e posteriormente, a parte radicular do elemento 21 foi novamente seccionada em duas partes, com o intuito de facilitar sua remoção. Os dentículos foram removidos um de cada vez, de acordo com a facilidade de saída, com auxílio de uma alavanca Apexo 303 (QUINELATO® - Schobell Industrial Ltda, Rio Claro - SP), aplicada vestibularmente e efetuado os movimentos de cunha, sarilho e alavanca, e de uma pinça hemostática curva (QUINELATO ${ }^{\circledR}$ Schobell Industrial Ltda, Rio Claro - SP) facilitando a retirada dos dentículos e fragmentos dentários da loja cirúrgica (Figura 3). Foram removidos 10 dentículos, e uma massa de consistência firme 
contendo em seu interior 04 dentículos, semelhante a capsula da lesão e foram acondicionados em formol a $10 \%$ e enviados para laudo histológico (Figura 4). Após a remoção de todos os fragmentos e elementos, executou-se a curetagem da loja cirúrgica, para remoção de todo o tecido patológico e regularização da loja óssea com cureta do tipo Lucas e lima para osso, respectivamente (QUINELATO ${ }^{\circledR}$ - Schobell Industrial Ltda, Rio Claro - SP).

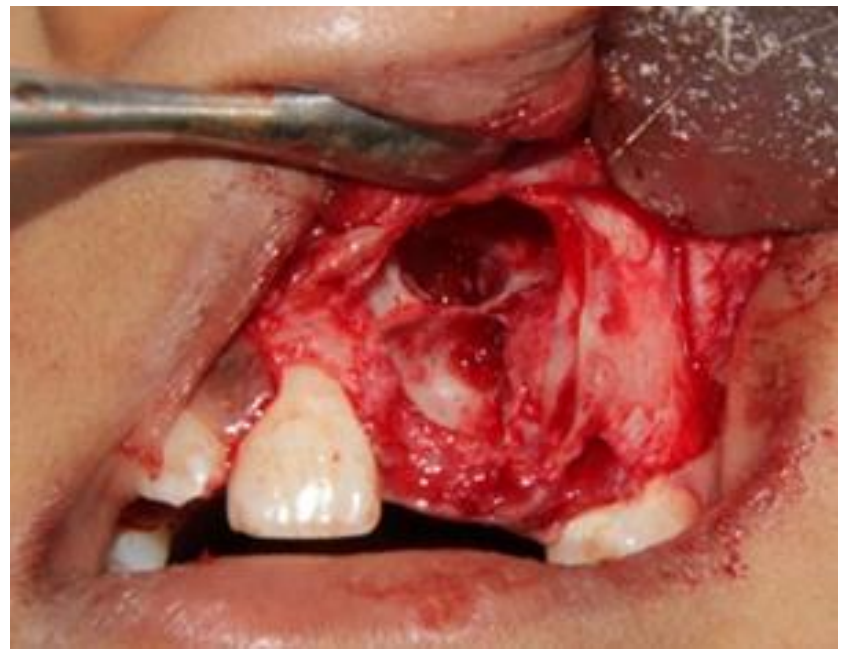

Figura 3: Aspecto trans-operatório após remoção da lesão.

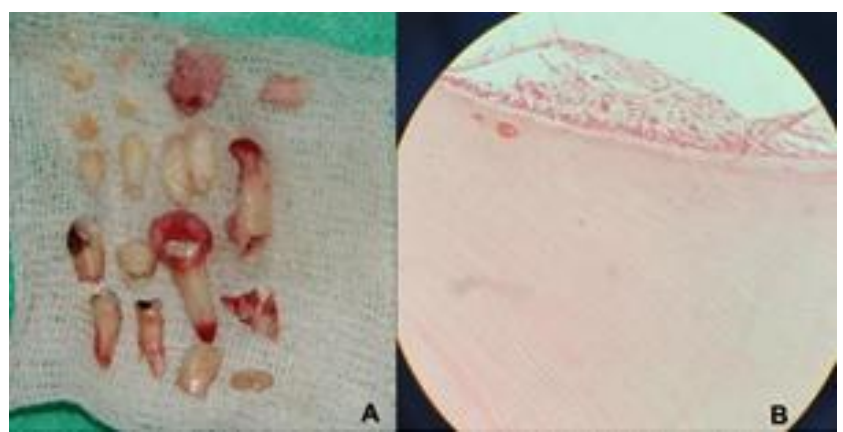

Figura 4: A - Espécime cirúrgico dos dentículos e fragmentos dentários do odontoma composto. B - Lâmina da lesão durante laudo histológico.

A limpeza da região foi realizada por meio de irrigação abundante com soro fisiológico $(0,9 \%)$. A síntese da ferida cirúrgica foi estabelecida com o auxílio de um fio de Nylon 4-0. O paciente foi orientado por escrito e verbalmente sobre os cuidados no período pós-operatório e prescrito analgésico (Dipirona 500mg de 6/6 horas durante três dias) e anti-inflamatório não esteroidal (Ibuprofeno $600 \mathrm{mg}$ de $6 / 6$ horas durante três dias) com intuito de um pós-operatório mais confortável e sem complicações. A peça foi enviada para exame histopatológico no Laboratório de Patologia da Universidade Federal de Campina Grande (UFCG), Patos, Estado da Paraíba, Brasil, onde foi comprovado o diagnóstico de odontoma composto em região anterior de maxila. No pós-operatório de um ano, o paciente evolui sem queixas clinicas. $O$ elemento 13 irrompeu na cavidade bucal e o elemento 23 encontra-se em fase de tracionamento ortodôntico (Figura 5).

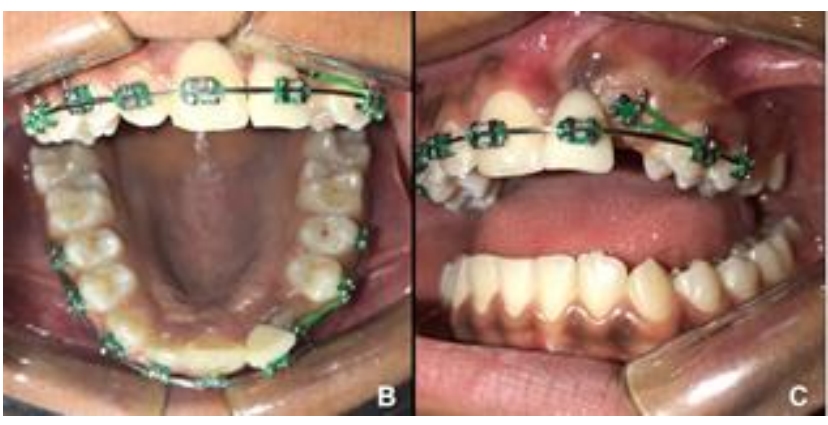

Figura 5: Pós-operatório de 1 ano. A - Vista oclusal. B - Vista vestíbulo-lateral.

\section{DISCUSSÃO}

Soluk Tekkesin et al. $^{7}$, em um estudo retrospectivo com crianças e adolescentes entre $0 \mathrm{e}$ 17 anos, observou que os cistos odontogênicos benignos (80\%), são mais frequentes quando comparados com os tumores odontogênicos benignos $(20 \%)$ e que a faixa etária mais acometida era a compreendida entre dos 13 aos 17 anos de idade $(63,1 \%)$, indo de encontro ao estudo de Abrahams e McClure $^{2}$ que apontam os tumores odontogênicos como raros. Soluk Tekkesin et al. $^{7}$ também concluíram que o tumor benigno mais comum na pediatria era o odontoma $(53,33 \%)$, seguido do ameloblastoma $(22,1 \%)$, corroborando com os estudos de Wang et al. ${ }^{8}$, Al-Khateeb et al. ${ }^{9}$, Guerrisi et al. ${ }^{10}$ e Lima et al. ${ }^{11}$.

Em relação à classificação histológica dos tumores odontogênicos, Abrahams e McClure $^{2}$ afirmam que podem ser classificados de acordo com seu tecido de origem em epitelial, mesenquimal e misto, mesma classificação pode ser observada no estudo de Bernardes ${ }^{12}$ e Silva ${ }^{13}$.

A etiologia dos odontomas é bastante complexa. Bereket et al. ${ }^{5}$, em estudos, sugere que fatores genéticos, trauma local e infecções podem estar associados ao aparecimento dos odontomas, mas sua etiologia permanece desconhecida. Owens et al. ${ }^{14}$ e Cildir et al. ${ }^{15}$ observam que, além desses fatores, a presença de dentes irrompidos esteja associado ao aparecimento dos odontomas, confirmando os achados em nosso paciente.

Angiero et al. ${ }^{3}$, Soluk Tekkesin et al. ${ }^{7}$ e Altay et al. ${ }^{16}$ destacam que os odontomas desenvolvem-se de forma assintomática, sendo diagnosticados por exames radiográficos de rotina, esfoliação dentária tardia ou posição ectópica de dentes permanentes, que acometem principalmente indivíduos do sexo masculino e que há uma predisposição para o aparecimento de odontoma composto em região anterior de maxila, características essas, observadas nesse relato. Porém, isso vai contra os achados do estudo de Freires $^{17}$, que relatou um caso de um odontoma composto em região anterior de mandíbula.

Segundo Neville et al. ${ }^{4}$ e Angiero et al. ${ }^{3}$, as manifestações clínicas mais comuns, são: alteração 
na erupção dos dentes permanentes quanto dos dentes decíduos, edema, agenesia de dentes permanentes, infecção, inflamação e mau posicionamento dental. Analisando o caso clínico descrito observou-se abaulamento ósseo, leve deslocamento dentário e alteração na erupção dentária, corroborando com os autores supracitados.

Kignel $^{18}$ ressalva a possibilidade de se estabelecer o diagnóstico do odontoma composto em função dos achados clínicos e radiográficos, pois seu aspecto de múltiplas estruturas radiopacas, semelhantes a dentes, circunscritas por um fino halo radiolúcido é bem característico, sendo essas características, observadas na análise radiológica do nosso paciente. Por outro lado, Bilodeau, Collins ${ }^{19}$ (2016) lista algumas lesões difíceis de serem distinguidas com base apenas na avaliação clínica e radiográfica, tornando-as diagnóstico diferencial dos odontomas, tais como: osteoma, fibroma ossificante, cementoblastoma, osteomielite esclerosante focal, tumor odontogênico cístico calcificante (TOCC) e fibro-odontoma ameloblástico.

A terapêutica escolhida no caso relatado, foi a enucleação da lesão, com a remoção dos elementos dentários a ela associados em razão de não apresentarem chances de aproveitamento, e está de acordo com os tratamentos propostos por Ladeinde et al. $^{20}$, Chang et al. $^{21}$ e Vázquez-Diego et al. $^{22}$. Importante observar que o estudo de Teruhisa et al. ${ }^{23}$ aponta que o tratamento do odontoma pode ser feito sem a remoção dos dentes adjacentes, fato esse não ocorrido em nosso caso.

\section{CONCLUSÃO}

Pode-se concluir que o tratamento proposto foi eficaz e que a tomografia computadorizada por feixe cônico é um exame complementar de grande valia no diagnóstico de patologias, assim como no planejamento cirúrgico, devido à alta resolução e precisão das imagens obtidas.

\section{REFERÊNCIAS}

1. Jayam C, Bandlapalli A, Patel N, Choudhary RS. A case of impacted central incisor due to dentigerous cyst associated with impacted compound odontome. BMJ Case Rep. 2014; 2014: bcr2013202447.

2. Abrahams JM, McClure SA. Pediatric Odontogenic Tumors. Oral Maxillofac Surg Clin North Am. 2016;28(1):45-58.

3. Angiero F, Benedicenti S, Parker S, Signore A, Sorrenti E, Giacometti E et al. Clinical and surgical management of odontoma. Photomed Laser Surg. 2014;32(1):47-53.

4. Neville BW, Damm DD, Allen CM, Bouquot JE. Patologia oral e maxilofacial. 4.ed. Rio de Janeiro: Elsevier, 2016.

5. Bereket C, Çakır-Özkan N, Şener İ, Bulut E, Tek M. Complex and compound odontomas: Analysis of 69 cases and a rare case of erupted compound odontoma. Niger J Clin Pract. 2015;18(6):726-30.

6. Malamed SF. Manual de anestesia local. 6. ed. Rio de Janeiro: Elsevier, 2013.

7. Soluk Tekkesin M, Tuna EB, Olgac V, Aksakallı $\mathrm{N}$, Alatlı C. Odontogenic lesions in a pediatric population: Review of the literature and presentation of 745 cases. Int J Pediatr Otorhinolaryngol. 2016;86:196-99.

8. Wang YL, Chang HH, Chang JY, Huang GF, Guo MK. Retrospective survey of biopsied oral lesions in pediatric patients. J Formos Med Assoc. 2009;108(11):862-71.

9. Al-Khateeb T, Al-Hadi Hamasha A, Almasri NM. Oral and maxillofacial tumours in north Jordanian children and adolescents: a retrospective analysis over 10 years. Int $\mathbf{J}$ Oral Maxillofac Surg. 2003;32(1):78-83.

10. Guerrisi M, Piloni MJ, Keszler A. Odontogenic tumors in children and adolescents. A 15-year retrospective study in Argentina. Med Oral Patol Oral Cir Bucal. 2007;12(3):E180-85.

11. Lima GS, Fontes ST, Araújo LMA, Etges A, Tarquinio SBC, Gomes APN. A survey of oral and maxillofacial biopsies in children: a singlecenter retrospective study of 20 years in PelotasBrazil. J Appl Oral Sci. 2008;16(6):397-402.

12. Bernardes VF, Cota LOM, Costa FO, Mesquita RA, Gomez RS, Aguiar MCF. Gingival peripheral odontoma in child: case report of an uncommon lesion. Braz J Oral Sci. 2008;7(26):1624-26.

13. Silva AR, Carlos-Bregni R, Vargas PA, de Almeida OP, Lopes MA. Peripheral developing odontoma in newborn. Report of two cases and literature review. Med Oral Patol Oral Cir Bucal. 2009;14(11):e612-15.

14. Owens BM, Schuman NJ, Pliske TA, Culley WL. Compound composite odontoma associated with an impacted cuspid. J Clin Pediatr Dent. 1995; 19(4):293-95.

15. Cildir SK, Sencift K, Olgac V, Sandalli N. Delayed eruption of a mandibular primary cuspid associated with compound odontoma. J Contemp Dent Pract. 2005;6(4):152-59.

16. Altay MA, Ozgur B, Cehreli ZC. Management of a compound odontoma in the primary dentition. $\mathrm{J}$ Dent Child. 2016;83(2):98-101.

17. Freires JFV. Remoção cirúrgica de odontoma composto de grande proporção sob anestesia local: relato de caso [monografia]. Patos (PB): Curso de Bacharelado em Odontologia, Universidade Federal de Campina Grande; 2017.

18. Kignel S. Estomatologia - bases do diagnóstico para o clínico geral. 2.ed. São Paulo: Santos; 2013.

19. Bilodeau EA, Collins BM. Odontogenic Cysts and Neoplasms. Surg Pathol Clin. 2017;10(1):177-22.

20. Ladeinde AL, Ajayi OF, Ogunlewe MO, et al. Odontogenic tumors: a review of 319 cases in a 
Nigerian teaching hospital. Oral Surg Oral Med Oral Pathol Oral Radiol Endod. 2005;99(2):191-95.

21. Chang JY, Wang JT, Wang YP, Liu BY, Sun A, Chiang CP. Odontoma: a clinicopathologic study of 81 cases. J Formos Med Assoc. 2003;102(12):876-82.

22. Vázquez-Diego J, Gandini Pablo C, Carbajal Eduardo E. Odontoma compuesto: Diagnóstico radiográfico y tratamento quirúrgico de um caso clínico. Av Odontoestomatol. 2008;24(5):307-12.

23. Teruhisa U, Murakami J, Hisatomi M, Yanagi Y, Asaumi J. A case of unerupted lower primary second molar associated with compound odontoma. Open Dent J. 2009;3:173-76.

\section{CONFLITO DE INTERESSES}

Os autores declaram não haver conflitos de interesse.

\section{AUTOR PARA CORRESPONDÊNCIA}

\section{Julierme Ferreira Rocha}

juliermerocha@ hotmail.com

Universidade Federal de Campina Grande, Centro de Saúde e Tecnologia Rural. Av. dos Universitários, s/n, Rodovia Patos/Teixeira, Km1, Bairro Jatobá,. 58708-110 - Patos, PB, Brasil

Telefone: (83) 3511-3045

Submetido em 10/05/2019

Aceito em 28/08/2019 